\title{
Brillouin frequency shift of standard optical fibers set in water vapor medium
}

\author{
Carlos Galindez, ${ }^{*}$ Francisco J. Madruga, and Jose M. Lopez-Higuera \\ Photonics Engineering Group, Universidad de Cantabria, Avenida Los Castros sn, Santander, 39005, Spain \\ *Corresponding author: galindezca@unican.es
}

Received July 23, 2009; revised November 6, 2009; accepted November 8, 2009; posted December 2, 2009 (Doc. ID 114483); published December 24, 2009

\begin{abstract}
The dependence of the Brillouin frequency shift (BFS) on UV-cured acrylate coating and uncoated fibers for media that have different water vapor concentrations is experimentally investigated. The BFS is proportional to the temperature within the fiber, but it also depends on the water vapor contained in the surroundings of the fiber. A hypothesis based on the efficiency of the heat transfer due to the different humidity concentration in the media is proposed, and the temperature difference that depends on the heat transfer is quantified in standard fibers. A shift of $\sim 0.22 \mathrm{MHz}$ for relative humidity change between $60 \%$ and $98 \%$ at $20^{\circ} \mathrm{C}$ is measured. (C) 2009 Optical Society of America

OCIS codes: $060.0060,060.2310,290.5830,290.5900$.
\end{abstract}

The measurement of environmental variables such as temperature or strain can be made using the dependence of the Brillouin frequency shift (BFS) on those variables $[1,2]$. This effect has been used to develop Brillouin-based fiber sensors for smart materials and structures [1] in environmental and seasonal conditions. The BFS is defined by the physical characteristics of the fiber that determine the propagation and the acoustic modes of the light. Thus the BFS can sense modifications in the material that are linked to thermal and force variations into the fiber.

The influence of relative humidity $(\mathrm{RH})$ on the measurement of the BFS, while the environmental temperature is controlled and kept constant, has been previously reported [3,4]. In this Letter, an explanation to such dependence and the setting of a possible uncertainty in the measurement of environmental temperature based on the heat transfer in the presence of water vapor is proposed. In the next paragraphs, the experimental setup is described; a hypothesis that explains this effect is proposed, and experimental data with their discussions are shown.

In standard optical fibers, the BFS is given by $\nu_{B}$ $=2 n_{\mathrm{eff}} V_{a} / \lambda_{p}$ [2], where $n_{\mathrm{eff}}$ is the effective refractive index of the fiber, $V_{a}$ is the effective longitudinal acoustic velocity, and $\lambda_{p}$ is the optical wavelength pump. In the stimulated Brillouin scattering, the phonon generated by the pump and the Stokes waves via the electrostriction process induces periodic pressure differences or refractive index changes in the fiber that act like a Bragg grating. Then measurements of the same environmental variable with Brillouin and Bragg grating techniques are useful to study the variations of the $n_{\text {eff }}$ in the BFS. The Bragg wavelength $\lambda_{\text {Bragg }}$ or resonance condition of a grating, is given by $\lambda_{\mathrm{Bragg}}=2 n_{\mathrm{Beff}} \Lambda$, where $n_{\text {Beff }}$ is the effective refractive index of the fiber Bragg grating (FBG) and $\Lambda$ is the grating pitch. The value of BFS depends on the $n_{\text {eff }}$ of the media and the $V_{a}$, while in the FBG the Bragg wavelength shift (BWS) depends on the refractive index and the grating pitch. However, variations in the pitch are negligible with no strain conditions.

As a first approach to clarify the effects of humidity in the measurement of BFS, a total dependence on the $n_{\text {eff }}$ is assumed. To obtain experimental data of the BFS and BWS, we used the typical setup shown in Fig. 1. Fiber 1 (F1) has a UV-cured acrylate coating and Fiber 2 (F2) is without coating; both are SMF28e fibers from the same reel and have $10 \mathrm{~m}$ of length. As a parallel setup to measure and identify how the refractive index behaves with variations of $\mathrm{RH}$, an FBG (with pitch of $1071.72 \mathrm{~nm}$ ) is written in an uncoated fiber section from the same spool of F1 and F2, assuring the same physical and geometrical conditions in the three fibers under test.

The influence of the fiber coating in the measurement of BFS for variations of $\mathrm{RH}$ is investigated before analyzing the refractive index behavior under the same circumstances. BFS in F1 and F2 for similar environmental conditions, and variations in the $\mathrm{RH}$, is measured. We found that the only effect caused by the coating is an offset in the BFS value with respect to the uncoated fiber. This offset is measured in $\sim 0.6 \mathrm{MHz}$ at $25^{\circ} \mathrm{C}$ (Fig. 2), which is related to the change of BFS caused by the thermal strain in coated fibers reported in $0.005 \mathrm{MHz} /{ }^{\circ} \mathrm{C}$ [5]. Besides, the fiber coatings made of UV-cured polymers have a water diffusion coefficient from $10-200 \mu \mathrm{gm} / \mathrm{m}^{2} \mathrm{~s}$

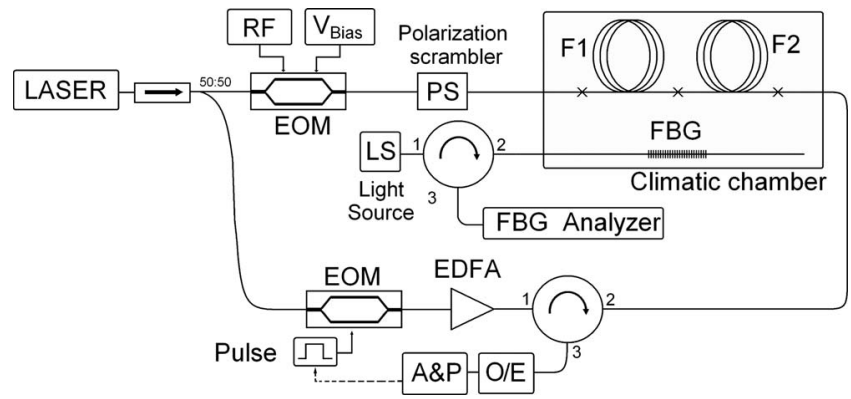

Fig. 1. Experimental setup. EOM, Electro-optic modulator; A\&P, analysis and processing; EDFA, erbium-doped fiber amplifier; $\mathrm{O} / \mathrm{E}$, optical-to-electrical converter. 


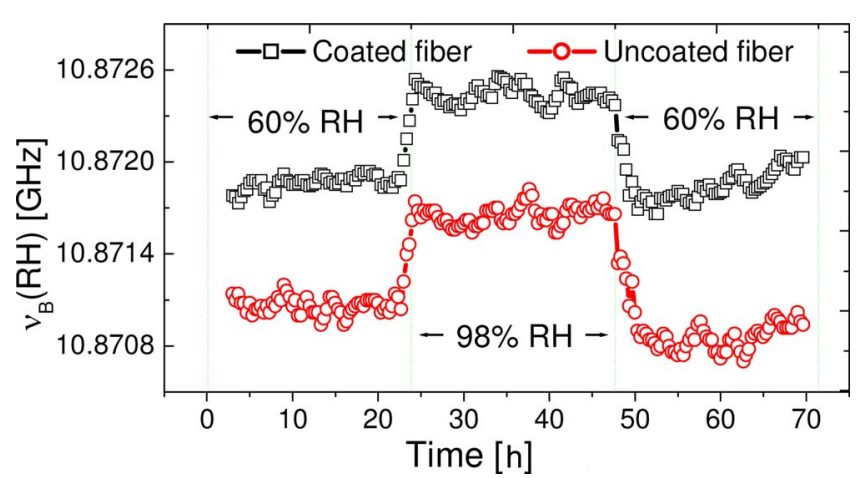

Fig. 2. (Color online) Experimental data for $\mathrm{RH}$ of $60 \%$ and $98 \%$. squares, coated fiber; circles, uncoated fiber.

[6]; thus the coating is rather highly permeable for water; i.e., the coating does not influence the variation of BFS with the relative humidity.

Once the coating effect is clarified, we proceed to measure the BWS and the BFS in the segments of uncoated fiber inside the climatic chamber. The data obtained for $25^{\circ} \mathrm{C}$ and $\mathrm{RH}$ states of $90 \%$ and $60 \%$ are plotted in Fig. 3. It can be noticed that at a constant temperature, the BWS responds similarly to changes of $\mathrm{RH}$, as does the BFS. It is also important to point out that the temperature is controlled and is measured with a precision of $0.2^{\circ} \mathrm{C}$; this value is smaller than the reported as shift caused by the $\mathrm{RH}$ variations. Besides, with the FBG we can demonstrate that the variation in BFS does not depend on an error or the uncertainty of the Brillouin temperature sensor neither does on the fiber coating, because the FBG is separately measured with another temperature sensor. Additionally, it is shown that the optical wave guide is the one that experiences these humidity conditions.

By measuring the BWS and assuring the nonstrain conditions in the fiber segment, the $n_{\text {Beff }}$ can be quantified as a function of the relative humidity. Then the variations on the BWS with the humidity may be attributed exclusively to alterations on the refraction index of the fiber. If in the calculation of $\nu_{B}, V_{a}$ is assumed as a constant the variations due to $\mathrm{RH}$ are exclusively attributed to $n_{\text {eff. }}$ Thus the difference of $n_{\text {Beff }}$ between two RH states $\left(\Delta n_{\text {Beff }}=n_{\text {Beff2 }}-n_{\text {Beff1 }}\right)$ and $n_{\text {eff }}$ in the same RH states $\left(\Delta n_{\text {eff }}=n_{\text {eff2 }}-n_{\text {eff1 }}\right)$ can be considered as equal. However, by using the experimental
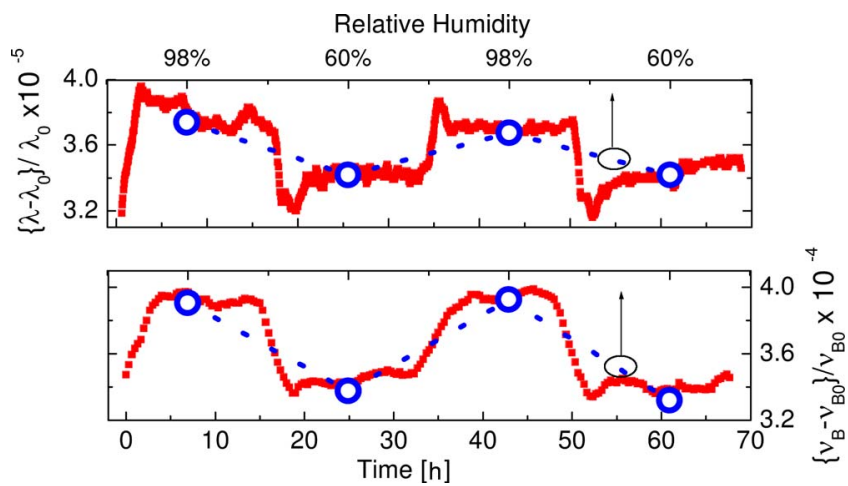

Fig. 3. (Color online) BWS (up) and BFS (down) measured under the same conditions. $\nu_{B 0}$ and $\lambda_{0}$ are the BFS and the BWS at $20^{\circ} \mathrm{C}$ and $50 \%$ of $\mathrm{RH}$.

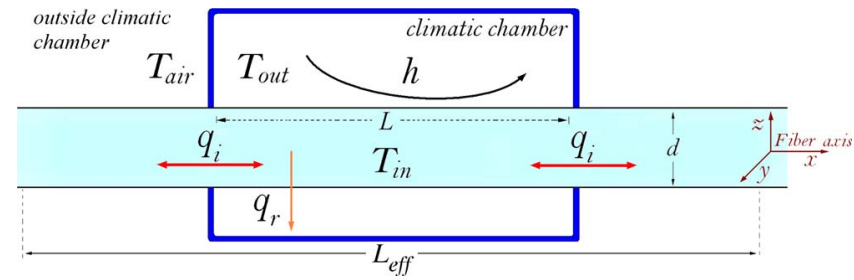

Fig. 4. (Color online) Scheme of thermal interaction of the fiber.

data, the values of $\Delta n_{\text {eff }}$ are almost 20 times greater

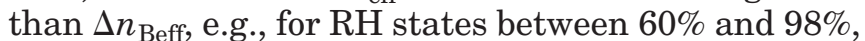
$\Delta n_{\text {eff }}$ and $\Delta n_{\text {Beff }}$ are found to be $6.685 \times 10^{-5}$ and $3.732 \times 10^{-6}$. So we can not attribute the variation of $\nu_{B}$ with the RH only to $n_{\text {eff }}$ or $V_{a}$ separately.

The experiment is an isothermal and an isochoric process. Thus the pressure and the mass of the mixture of air and water vapor are nonconstant variables. If the cause of this change is the air pressure in the climatic chamber, a variation of $0.5 \mathrm{MHz}$ in the BFS should be equivalent to $\sim 5.5 \mathrm{MPa}$, using -91 $\times 10^{-6} \mathrm{kHz} / \mathrm{Pa}$ [7]. However, the pressure difference when the air goes up from $60 \%$ to $98 \% \mathrm{RH}$ is $\sim 4.6 \mathrm{kPa}$. A similar case occurs in the FBG, where the pressure sensitivity of the fractional change in Bragg wavelength is $-2.02 \times 10^{-6} \mathrm{MPa}[1,8]$. According to the experimental data the BWS is 3 orders of magnitude smaller than the expected value for pressure variations. Besides, the more water vapor mass in the air, the more air pressure, which means a BFS drop in frequency. Looking at Figs. 2 and 3 we can notice that BFS and BWL go up in the frequency spectra and are not decreasing as in the case of pressure. In consequence, the air pressure is not the cause of this behavior.

Although the BFS and BWS are concomitant effects, their measurements are under the same environmental situation; the measurement of temperature and difference of temperature $(\Delta T)$ with both methods are the same. This may be expressed in terms of the temperature coefficient $C_{T}$, the photoelastic constant of the fiber $P_{e}$, the fiber thermalexpansion coefficient $\alpha$, and the fiber-thermo-optic coefficient $\xi$ [1] by (for nonstrain conditions)

$$
\frac{\Delta \nu_{B}}{C_{T}}=\Delta T_{B}=\Delta T_{\text {Bragg }}=\frac{\Delta \lambda_{\text {Bragg }}}{\lambda_{\text {Bragg }}} \frac{1}{\left[\left(1-P_{e}\right) \alpha+\xi\right]} .
$$

According to Eq. (1) we can compare measurements of $\Delta T$ measured with Bragg and Brillouin sensors. Then we focus our discussion in the implications

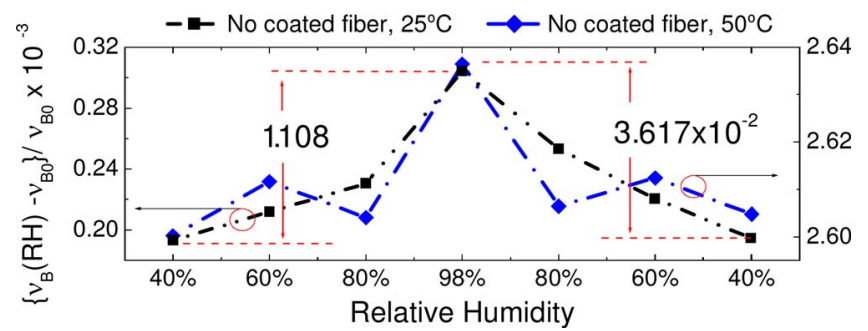

Fig. 5. (Color online) BFS as a function of $\mathrm{RH}$ for $T_{\text {out }}$ of $25^{\circ} \mathrm{C}$ (squares) and $50^{\circ} \mathrm{C}$ (diamonds). 
Table 1. Summary of Experimental Data

\begin{tabular}{ccccccccc}
\hline & \multicolumn{4}{c}{$25^{\circ} \mathrm{C}$} & \multicolumn{4}{c}{$50^{\circ} \mathrm{C}$} \\
\cline { 2 - 9 } & $40 \%$ & $60 \%$ & $80 \%$ & $98 \%$ & $40 \%$ & $60 \%$ & $80 \%$ & $98 \%$ \\
\hline Abs. humidity $\left(\mathrm{g} / \mathrm{m}^{3}\right)$ & 8.13 & 11.9 & 16.02 & 19.8 & 31.81 & 49.2 & 67.23 & 81.1 \\
Air pressure (Pa) & 1263 & 1894 & 2543 & 3133 & 4935 & 7424 & 9876 & 14559 \\
$\Delta \nu_{B} / \nu_{B} \times 10^{-4}$ (uncoated) & 1.93 & 2.12 & 2.31 & 3.04 & 26.0 & 26.12 & 26.04 & 26.36 \\
$\Delta \lambda_{B} / \lambda_{B} \times 10^{-5}(\mathrm{FBG})$ & 2.7991 & 3.1597 & 3.2689 & 3.5691 & 19.209 & 19.261 & 19.221 & 19.303 \\
\hline
\end{tabular}

of water vapor mass as a function of the absolute humidity in the media. The relative humidity is the measure of the amount of water vapor contained in the air at a particular temperature. The capacity of air to hold water vapor varies according to the temperature of the air. The system that comprises the fiber, the climatic chamber, and environment conditions outside the climatic chamber is depicted in Fig. 4. Taking into consideration the heat convection and heat exchange, the equation for thermal transfer [9] without radiation may be solved as follows:

$$
\left(T-T_{\text {out }}\right)(t)=\left(T_{0}+\frac{q_{i} d}{4 h}\right) e^{-t 4 h / d \rho c}-\frac{q_{i} d}{4 h}\left(\frac{L_{\text {eff }}}{L}\right),
$$

where $h$ is the convection heat-transfer coefficient, $\rho$ is the density of the fiber, $q_{i}$ is the heat exchange rate, $L_{\text {eff }}$ is the effective length of fiber in the total heat transfer process (inside and outside the chamber), and $d$ is the fiber diameter.

The first term on the right side of Eq. (2) describes evolution in time and stabilization of thermal system variables, which are assumed stable in each "state of relative humidity," since the elapsed time allows the variables to reach stabilization. There is also a previous transition state where the variables stop changing. This outcome allows a single temperature and pressure to be attributed to the whole system. However, the second term in Eq. (2) predicts a small temperature value, which depends on the efficiency of heat transfer in the system, the length of fiber outside the chamber, and the quantity of heat that the fiber exchanges outside the climatic chamber. The specific heat capacity of water vapor is $1.84 \mathrm{~kJ} / \mathrm{kg} \mathrm{K}$. Thus the higher vapor concentration, the better the heat transfer, or vice versa. $60 \% \mathrm{RH}$ at $25^{\circ} \mathrm{C}$ is equivalent to $11.9 \mathrm{~g} \mathrm{~m}^{-3}$ of water vapor in the air. If the amount of $\mathrm{RH}$ is increased up to $98 \%$, the water vapor is $19.8 \mathrm{~g} \mathrm{~m}^{-3}$, so the heat transfer is more efficient in the second state than in the first one, which means that the temperature into the fiber is similar to $T_{\text {out }}$.

According to our previous discussion, if the temperature is higher absolute humidity is also higher; then a better heat transfer efficiency in the system makes that the temperature into the fiber looks more like $T_{\text {out }}$. The higher the temperature, the lesser the difference between humidity states (see Fig. 5). Using data of Table 1 and $P_{e} @ 0.22$ [1], we found that a BWS of $\sim 5 \times 10^{-3} \mathrm{~nm}$ for $\mathrm{RH}$ variations from $60 \%$ to
$98 \%$ corresponds to a $\Delta T$ of $\sim 0.48^{\circ} \mathrm{C}$. In the case of the uncoated fiber, the normalized thermal responsivity at a constant strain is $6.67 \times 10^{-6}{ }^{\circ} \mathrm{C}$ [1]. With a measured $C_{T}$ of $0.92 \mathrm{MHz} /{ }^{\circ} \mathrm{C}$, the BFS is $0.45 \mathrm{MHz}$ and is equivalent to $0.488^{\circ} \mathrm{C}$.

Summing up, the effects of water vapor concentrations in the environmental media on BFS in an optical fiber with and without UV-cured acrylate coating were experimentally investigated. We found that the reported pattern in BFS as a function of relative humidity also appears in the Bragg grating as the $\mathrm{RH}$ varies. The shift due to the thermo-optic effect fits with the BFS for relative humidity changes at a constant temperature. The BFS depends on the water vapor concentration in air and on the temperature. At a high $\mathrm{RH}$ the absolute humidity increases, and the fiber reaches more efficiently the set temperature; hence the variation in BFS depends on the effective heat transfer between the air-silica or airjacket-silica media. The measurement accuracy of the environmental temperature has to include the uncertainty caused by humidity in a Brillouin temperature sensor. For a SMF28e fiber we measured this uncertainty in a range that goes from $0.15^{\circ} \mathrm{C}$ to $0.5^{\circ} \mathrm{C}$ according to the $\mathrm{RH}$ percentage and the air temperature.

Financial support for this work was provided by the Spanish Ministry of Science and Technology via the grant BES-2005-8287 of TEC2007-67987-C02-01 project.

\section{References}

1. J. López-Higuera, ed., Handbook of Optical Fibre Sensing Technology (Wiley, 2002).

2. G. P. Agrawal, Nonlinear Fiber Optics, 3rd ed. (Academic, 2001).

3. C. Galindez, F. Madruga, M. Lomer, A. Cobo, J. M. Lopez-Higuera, presented at the 19th International Conference on Optical Fiber Sensors, April 14-18, 2008, Perth, Australia.

4. C. Galindez, F. Madruga, and J. M. Lopez-Higuera, IEEE Photonics Technol. Lett. 20, 1959 (2008).

5. T. Kurishima, T. Horiguchi, and M. Tateda, Appl. Opt. 29, 2219 (1990).

6. J. Rayss, W. M. PodkoCielny, and J. Widomski, J. Appl. Polym. Sci. 49, 835 (1993).

7. S. L. Floch and P. Cambon, Opt. Commun. 219, 395 (2003).

8. M. G. Xu, L. Reekie, Y. T. Chow, and J. P. Dakin, Electron. Lett. 29, 398 (1993).

9. F. Incropera, Fundamentals of Heat and Mass Transfer, 2nd ed. (Wiley, 2002). 